\title{
Sicherheit und Ästhetik. Historische und kulturelle Aspekte einer aktuellen Kon- junktur
}

\author{
Johannes Endres
}

\begin{abstract}
Der Beitrag plädiert für eine ,Abrüstung' und Öffnung des Diskurses über Sicherheit. Dabei sollen jedoch politisch-technische und kulturelle Aspekte des Begriffs nicht gegeneinander ausgespielt werden. Vielmehr soll deren historische Genese aus einer Gemengelage der Probleme zwischen Früher Neuzeit und frühem 19. Jahrhundert deutlich werden. Als Ansatzpunkt dienen Beispiele der Theoretisierung von Unsicherheit in den unterschiedlichen Epistemen (der Politik, der mathematischen und der ästhetischen Wahrscheinlichkeit, des Risikos usw.) sowie der Medialisierung des Terrors im Diskurs über das ,Erhabene' seit Hobbes. Insbesondere die Erhabenheitstheorie hat aufschlussreiche Konzepte von ,innerer' und ,äußerer' Sicherheit entwickelt, deren Wandel und deren Zusammenhang mit politisch-technischen Sicherheitskonzepten angedeutet werden soll. Dabei zeigt sich auch, dass die gegenwärtige Krise einer ,symmetrischen' Sicherheitsarchitektur nicht nur eine neue Qualität der Bedrohung, sondern auch traditionelle psychosoziale Reaktionsmuster zeitigt. Folglich geht es um zweierlei: darum, die Geschichtlichkeit unseres Sicherheitsbedürfnisses aufzuzeigen, und darum, aktuelle Befindlichkeiten umgekehrt auf ein in ihnen mitlaufendes kulturelles Gedächtnis der Ästhetik und der Kunst zurück zu beziehen. Angesichts dieser Aufgabe kann der Beitrag lediglich Vorüberlegungen bieten.
\end{abstract}

Keywords: Sicherheit/Unsicherheit; Wahrscheinlichkeit; Risiko; Schrecken/Terror; Ästhetik des Erhabenen

\section{Sicherheit als kulturelles Paradigma}

Die gegenwärtige Debatte über Sicherheit und Unsicherheit ist weitgehend auf politische und technische Aspekte des Themas konzentriert. Mit einer solchen Einschränkung steht sie in einer Diskurstradition, die keinesfalls älter - aber auch nicht jünger - als rund 500 Jahre ist. Ihr Beginn lässt sich - für den europäisch-abendländischen Kulturraum mit der Herausbildung des frühneuzeitlichen absolutistischen Staates und mit Hobbes' Begründung der Staatsräson über den Sicherheitszweck datieren (vgl. Conze 1994): „Die Aufgabe des Souveräns, ob Monarch oder Versammlung, ergibt sich aus dem Zweck, zu dem er mit der souveränen Gewalt betraut wurde, nämlich der Sorge für die Sicherheit des Volkes“ (Hobbes 1991, 255). Zu den Voraussetzungen einer solchen Verknüpfung von Sicherheit und politisch-technischer Sphäre gehört zum einen die historische Unsicherheitserfahrung der Bürgerkriege des 16. und 17. Jahrhunderts und zum anderen ein post- oder spät-konfessionelles Menschenbild, das die Sozialdisziplinierung der Untertanen nicht mehr an eine außerweltliche Autorität bindet, sondern dem weltlichen Souverän - dem „sterblichen Got[t]“ (Hobbes 1991, 134) - bzw. der Selbstformierung des Individuums unterstellt. Dieser semantische und bewusstseinsgeschichtliche Prozess ist - aus unterschiedlicher Perspektive - von Franz-Xaver Kaufmann (1973), Andrea Schrimm- 
Heins (1991/92), Michel Foucault (2004) u.a. dargestellt worden. Die Konsequenzen einer solchen Diskursivierung sind im Einzelnen vielgestaltig, lassen sich aber auf lange Sicht - und je nach Methodenstandpunkt - als Säkularisierung und Immanentisierung, als Pragmatisierung und Verinnerlichung sowie als zunehmende Selbstreferentialisierung des Sicherheitsbegriffs resümieren. Zwei Tendenzen scheinen dabei mit Blick auf unsere Fragestellung besondere Aufmerksamkeit zu verdienen: erstens die Positivierung von Sicherheit als individueller und gesellschaftlicher, Wertidee' sowie ihre Hinordnung auf den Bereich menschlicher Handlungskompetenz und zweitens die Problematisierung von Sicherheit als permanent gefährdetem oder immer schon verlorenem materiellem oder moralischem Gut, das sich in dem Maße zu verknappen droht, in dem das Sicherheitsinteresse ausgeweitet und verabsolutiert wird - eine Tendenz, durch die sich Sicherheit zunehmend darauf reduziert, die Abwesenheit ihres Gegenteils zu bezeichnen und zu organisieren. Beide Diskurskoordinaten kommen in den folgenden Zitaten der Forschung zum Ausdruck: „Sicherheit kann zum Wertbegriff nur unter den Bedingungen eines Weltverständnisses werden, in dem der Mensch und nicht mehr Gott als der eigentlich Handelnde, als der ,Geschichte Machende’ verstanden wird“ (Kaufmann 1973, 54) - „Auf Grund zahlreicher Hinweise [...] darf die These gewagt werden, daß für das moderne Bewußtsein Sicherheit überhaupt nur als Nicht-Unsicherheit positiv bestimmt werden kann“ (Kaufmann 1973, 158). Mit einer solchen Bilanz sind wesentliche Prämissen des ,Sicherheitsdispositivs' (Foucault) bis in die Gegenwart bezeichnet, deren historische Position sich umgekehrt als diskursgeschichtliches Ergebnis neuzeitlicher Kodierungen zu erkennen gibt. Zweierlei macht ein solcher genetischer Befund wiederum bewusst: dass (und inwieweit) ein aktueller Sicherheitsbegriff von historischen Programmierungen abhängig ist und dass Sicherheit weder als überkulturelle Universalie noch als anthropologisches Grundbedürfnis generalisiert werden kann. In methodischer Hinsicht vermag den Problemen des Sicherheitskonzepts daher nur eine ,Abrüstung' gerecht zu werden, die Sicherheit als „,breitere Konstruktion“ (Bonß 1995, 18) und - nicht zuletzt - als kulturelles Paradigma begreift, das in seinen Formatierungen und Anschlussoperationen auf vielfältige Weise mit einer materiellen und politischen Sicherheitssemantik interferiert. ${ }^{1}$

\section{Sicherheit, Wahrscheinlichkeit, Risiko}

An der Technisierung und Operationalisierung des Sicherheitsbegriffs ist in erheblichem Maße auch eine terminologische Umstellung beteiligt, deren Anfänge bis ins 17. Jahrhundert zurückverfolgt werden können. Sie ist - im weitesten Sinne - einem Versuch der Unsicherheitsprävention geschuldet, der auf einen prinzipiellen Aspekt der Sicherheitsintransparenz, denjenigen der „Zukunftsungewißheit“ (Luhmann 1991, 16) reagiert. Es handelt sich - mit einer Formulierung Hans Blumenbergs - um den Prozess der ,Terminologisierung der Wahrscheinlichkeit' (Blumenberg 1998). Dieser ist vom doppelten Bewusstsein der Unsicherheit der Zukunft und der gleichzeitigen Notwendigkeit eines

1 Die vorliegenden Überlegungen berühren sich in wesentlichen Punkten mit meinen Darlegungen in Endres (2008). Dort finden sich auch ausführlichere Erläuterungen zu den hier nur verkürzt behandelten Punkten. 
Kontingenzarrangements bestimmt. Wahrscheinlichkeit wird dabei nicht mehr - wie in weiten (wenn auch nicht allen) Teilen der antiken, mittelalterlichen und frühneuzeitlichen Tradition - über eine Entgegensetzung von ,Wahrscheinlichkeit und Wahrheit' oder ,Wahrscheinlichkeit und Wirklichkeit' formalisiert, sondern auf den Erwartungshorizont der Zukunft ausgerichtet und zum Index einer ,Ereigniswahrscheinlichkeit', die ihrerseits (mehr oder weniger exakt) quantifiziert und objektiviert werden kann (vgl. Buldt et al. 2004). Eine solche „Logisierung und Kalkülisierung der ,Wahrscheinlichkeit'“ (Blumenberg 1998, 130) - bei Pascal, Huygens, Jacob und Niklaus Bernoulli u.a. - wird v.a. auf den Gebieten des Glückspiels und der Statistik, also der Wahrscheinlichkeitsberechnung a priori und a posteriori ventiliert. Eine ihrer wichtigsten Folgen (im hier interessierenden Zusammenhang) ist, dass Wahrscheinlichkeit und Unwahrscheinlichkeit nicht mehr als Oppositionsbegriffe zu Gewissheit und Sicherheit gefasst, sondern als deren technischmathematischer Ausdruck verstehbar werden. Damit muss freilich auch die Leitdifferenz von Sicherheit/Unsicherheit aufgegeben werden, da die Zukunft immer unsicher und allenfalls ein ,Restrisiko' präzisierbar ist. Der Sicherheitsdiskurs wird damit entschieden nach vorne geöffnet und ,rationalisiert' zugleich: „Schäden sollen nach Möglichkeit vermieden werden. Da diese Maxime allein die Handlungsmöglichkeiten zu stark limitieren würde, muß man auch Handlungen zulassen, und das eben heißt: ,riskieren', die einen im Prinzip vermeidbaren Schaden verursachen können, sofern nur die Kalkulation der Schadenswahrscheinlichkeit und der etwaigen Schadenshöhe dies als vertretbar erscheinen läßt“" (Luhmann 1991, 22). ${ }^{2}$

Die Systemtheorie Niklas Luhmanns trägt einer solchen Ausdifferenzierung Rechnung, indem sie anstelle von Sicherheit/Unsicherheit die Unterscheidung von ,Risiko und Gefahr' etabliert, bei der das Interesse an Sicherheit zwar „vorausgesetzt“, aber nicht „markiert“" wird, da es nicht deren Gegenteil ist: der Gefahr nicht, weil es absolute Sicherheit nicht gibt, des Risikos nicht, weil Unsicherheit ohne Risiko unbegrenzbar bliebe (Luhmann 1991, 33). Ein so verstandenes Risiko-Dispositiv bedient sich stattdessen des seit dem 17. Jahrhundert evolvierten „Mediums der Wahrscheinlichkeit/Unwahrscheinlichkeit“, um die Unsicherheit der Zukunft für die je eigenen Zwecke zu antizipieren: „Mit der Form des Risikos nutzt man gerade die Unbestimmtheit der Zukunft, ja gleichsam die eigene Unwissenheit aus, um die Gegenwart auf Formen zu bringen, die durch künftige Gegenwarten bestätigt oder auch widerlegt werden können. Die Zukunft, die nur auf die eine oder andere, jedenfalls aber nur auf eine bestimmte Weise Gegenwart werden kann, wird in eine fiktive Form gebracht, die als solche nie eintreten wird, nämlich die Form wahrscheinlich/unwahrscheinlich“ (Luhmann 1991, 80f.). In diesem Sinne gibt es zwar das Bestreben, Gefahren, die der Umwelt angelastet werden, in Risiken zu transformieren, die der eigenen Entscheidungskompetenz zugeschrieben werden (vgl. Luhmann 1991, 54f.), aber keine Erfolgsgewissheit, da jede Entscheidung neue, prinzipiell ergebnisoffene Risiken produziert. ${ }^{3}$ Ein solcher Sprachgebrauch ist nicht nur selbst hochgradig

2 Eine solche Auffassung wird von Luhmann als ,rationalistisches Modell' bezeichnet, das es allerdings durch eine systemtheoretische Betrachtung im Sinne einer Beobachtung zweiter Ordnung abzulösen gelte (vgl. auch Luhmann 1991, 28f.).

3 Luhmann frei nach Hölderlin: „Wo aber Kontrolle ist / Wächst das Risiko auch“ (Luhmann 1991, 103). Oder auch: „Das Risiko ist die Gefahr“ (Luhmann 1991, 131). 
formalisiert, der Apparat der Risikokalkulation setzt die Errungenschaften der technischen Welt auch in instrumenteller Hinsicht voraus - so die Geometrisierung des Zufalls im neuzeitlichen Probabilismus, die Institutionen der Versicherungen usw. (vgl. Luhmann 1991, 54f.). Neben der Technologisierung der Sicherheit durch den neuzeitlichen Staat zählt die Pragmatisierung der Kontingenz durch die ,Risikogesellschaft' daher zu den wichtigsten Faktoren eines vorwiegend technisch konzipierten Begriffs der (Un)Sicherheit.

\section{Symmetrische und asymmetrische Sicherheitstechnik}

Die aktuellen Bedrohungen durch den transnationalen Terrorismus werden darum auch als Bedrohungen einer technisch-protektionistischen Sicherheitsarchitektur reflektiert. Dabei steht jedoch keineswegs nur der Sicherheitsapparat im engeren Sinne in Frage, sondern die westliche Sicherheitsdoktrin als solche, die sich im Gefolge der Bürgerkriege des 16./17. Jahrhunderts herausgebildet hat. Die Staatenkriege der Neuzeit - bis hin zu den ,regulären' Kriegen des 18. und 19. Jahrhunderts - haben die Sicherheit des Leviathans und des einzelnen Untertanen zwar gefährden, nicht aber den grundsätzlichen Geltungsanspruch einer Sicherheitsnorm bestreiten können. Deren wechselseitige Akzeptanz ist vielmehr ein wesentliches Fundament so genannter, symmetrischer' Sicherheitskonflikte gewesen, die dem Ziel der Wiederherstellung von Sicherheit dienten. Clausewitz' berühmtes Wort vom Krieg als der „Fortsetzung der Politik unter Einmischung anderer Mittel" erhebt eine solche Orientierung auch und gerade im Unsicherheitsfall zur allgemeinen Handlungsmaxime (Münkler 2006, 10). Aber bereits die Partisanenkriege des 19. Jahrhunderts stellen durch eine Strategie von Terror und Gegenterror und durch die Aufhebung der klaren Unterscheidung von Krieg und Frieden, Kombattant und NichtKombattant, Feind und Verbrecher, Militär und Zivil die finale Sicherheitspflichtigkeit politischen Handelns in Frage (Schmitt 1963). Eine solche Erosion droht grundsätzlich immer dann, wenn die ,innerstaatlichen wie [...] zwischenstaatlichen Begriffe von Regularität und Irregularität, Legalität und Illegalität“ sich nicht mehr ,,inhaltlich decken oder doch wenigstens in ihrer Struktur einigermaßen homogen sind" (Schmitt 1963, 41). Die neuere Forschung nennt solche Konstellationen , asymmetrisch', da in ihnen die Differenz von innerer und äußerer Sicherheit, von Krieg und Frieden, von Krieg und Bürgerkrieg sowie von politischen und ökonomischen Zielen kollabiert ist (Münkler 2006; Münkler 2007). Im Unterschied zum Partisanenkrieg wird der transnationale Terrorismus jedoch so bei Herfried Münkler - nicht als defensive, sondern als offensive Aggression definiert, die darüber hinaus entterritorialisiert, privatisiert und kommerzialisiert ist (Münkler 2006, 221ff.). Insofern lässt er sich mit dem „klassischen Verwüstungskrieg“ vergleichen, der ebenfalls durch eine Asymmetrie der Schwäche, eine verdeckte Konfrontation und wirtschaftliche Absichten gekennzeichnet ist. Daher kennt der Terrorismus auch kein primordiales Sicherheitsinteresse mehr noch ist er der Erreichung einer - verlorenen - symmetrischen Konfrontation verpflichtet (Münkler 2006, 230). Gerade dadurch aber treffen etwa Selbstmordattentäter nicht nur die politischen Sicherungssysteme des Westens, sondern auch das diesen vorausliegende individuelle wie kollektive „Sicherheitsempfinden“ 
(Münkler 2006, 345). Dabei bleibt natürlich zu bedenken, wie schon Carl Schmitt konstatiert, dass jeder Bestimmungsversuch des ,irregulären' (oder , ungehegten') Krieges - als Beobachtung zweiter Ordnung - „von der Kraft und Bedeutung des von ihm in Frage gestellten Regulären bestimmt ist" (Schmitt 1963, 11). Damit tangiert der terroristische Schrecken aber umgekehrt auch die sprachlich-begriffliche und symbolische Ordnung des westlichen Sicherheitsdenkens zentral.

\section{Schrecken und Terror: doppelte Diskurse}

Auch dem frühneuzeitlichen Staatsgebilde ist ,Terror' keineswegs fremd - und zwar nicht nur als feindlicher Schrecken, sondern auch als Instrument der Abschreckung, das wiederum repressiv gegen den schädlichen Schrecken eingesetzt wird. Beide Schreckenspotenziale werden in Hobbes Staatsphilosophie getrennt und hinsichtlich ihrer Verträglichkeit mit dem Sicherheitszweck des Staates geschieden: als Behemot-Schrecken, der die „Herrschaft der Leidenschaften, Krieg, Furcht, Armut, Häßlichkeit, Einsamkeit, Barbarei, Unwissenheit, Rohheit“ impliziert, und als Leviathan-Schrecken, der die „Herrschaft der Vernunft, Frieden, Sicherheit, Reichtum, Schmuck, Gemeinschaft, Glanz, Wissenschaft und Wohlwollen" nach sich zieht (Hobbes 1966, 175 [Vom Bürger, 10, 1]). Das Regime des einen Ungeheuers ist also gegen das des jeweils anderen gerichtet, Sicherheit aber das Ergebnis einer erfolgreichen Abschreckung des Behemot-Schreckens durch den Leviathan-Schrecken (vgl. Schmitt 1982, 33f.; Hobbes 1991, 134). Die Vorbildfunktion, die ein alttestamentlicher, rächender Gott für eine solche Auszeichnung des Terrors hat, ist offensichtlich: ${ }^{4}$ „Deshalb besitzen diejenigen, deren Macht unwiderstehlich ist, auf Grund ihrer überragenden Stellung oder Gewalt von Natur aus die Herrschaft über alle Menschen, und folglich liegt es an dieser Gewalt, daß das Königreich über die Menschen und das Recht, sie nach Belieben heimzusuchen, von Natur aus Gott dem Allmächtigen zusteht, und zwar nicht in seiner Eigenschaft als Schöpfer oder Gnadenspender, sondern als Allmächtiger“ (Hobbes 1991, 273). Daneben bringt die Positivierung des Schreckens bei Hobbes aber auch eine ästhetische Dimension hervor, sofern der Terror-Schaden nicht den Betrachter, sondern einen Anderen trifft - eine Konstellation, an die die moderne Ästhetik des Erhabenen und eine spezifisch ästhetische Theorie der Sicherheit anknüpfen können (vgl. Zelle 1987): „Fremdes Unglück zu sehen, ist etwas Angenehmes; denn es gefällt, nicht sofern es ein Unglück ist, sondern sofern es ein fremdes Unglück ist. Daher kommt es, daß Menschen zusammenlaufen, um sich Tod und Gefahren anderer anzusehen“ (Hobbes 1966, 26 [Vom Menschen, 11,12]). Im Laufe des 18. Jahrhunderts jedoch und verstärkt nach den historischen Erfahrungen des revolutionären Jakobinismus - werden der politische und der ästhetische Terror-Diskurs separiert: Der politische Schrecken wird abgewertet und als Herrschaftsmittel delegitimiert, während dem Schrecken im Medium der Kunst - vereinfacht gesprochen - eine positive Bedeutung inhärent bleibt. Letz-

4 Horst Bredekamp weist in seiner Untersuchung der Bildgeschichte des Leviathan-Frontispizes auf diese Tradition hin, die exemplarisch in der Inschrift der Weltgerichtsdarstellung im Tympanon von Autun zum Ausdruck kommt: „Terreat hic terror quos terrenus alligat error, nam fore sic verum notat hic horror specierum." (Bredekamp 2006, 160f.) 
teres erklärt sich u.a. aus einer kathartischen Funktionszuschreibung an die Kunst, wobei die ,Reinigung der Leidenschaften' mal als Immunisierung gegen den Schrecken, mal als Disziplinierung durch den Schrecken und mal als Optimierung des Schreckens zugunsten einer wertvollen Leidenschaft (wie z.B. ,Mitleid') ausgelegt wird (vgl. Zelle 2003, 437). Die Folgen einer solchen Ausdifferenzierung wirken bis in den heutigen Sprachgebrauch nach: So kann das deutsche Wort ,Schrecken' zwar das Fremdwort, terror' ersetzen, nicht aber umgekehrt, weil ,Schrecken' eine (prinzipiell positivierbare) ästhetische Konnotation besitzt, ,Terror' - das erstmals um 1795 in die deutsche Sprache eindringt - aber ausschließlich eine politische oder moralische Anti-Qualität transportiert (Walther 1990, $325)$.

\section{Edmund Burke: erhabene und schöne Kultur}

Edmund Burkes politische und kunsttheoretische Reflexionen markieren ziemlich genau den Umschlagspunkt einer solchen Entwicklung. In den Reflections on the Revolution in France von 1790 wird die jakobinische Schreckensherrschaft sowohl politisch als auch ästhetisch diskreditiert (Burke 1987, 167f.) - im Gegenzug erhebt Burke in A Philosophical Enquiry into the Origin of Our Ideas of the Sublime and Beautiful von 1757 den Schrecken zum „beherrschende[n] Prinzip des Erhabenen“ (Burke 1956, 92). Dabei ändert sich jedoch auch die Bedeutung der eigenen Sicherheit für die erhabene Delektion. Während diese für Hobbes noch als „Ursache“ der ästhetischen Empfindung fungiert, wird sie bei Burke zu deren „Bedingung“ zurückgestuft und Hobbes' schroffer Terrorvoyeurismus mit einer Ethik der Solidarität konfrontiert: „Ebenso sicher nun aber ist es unbedingt notwendig, daß mein Leben völlig außer jeder drohenden Gefahr sei, wenn ich über die Leiden anderer - über wirkliche oder eingebildete - oder wenn ich überhaupt über irgendeine Sache froh sein soll, - mag sie beruhen, worauf sie will. Aber es ist ein Fehlschluß, daraus zu folgern, die Freiheit von Gefahr sei in diesen oder irgendwelchen anderen Fällen die Ursache meines Frohseins. Niemand, glaube ich, kann in seinem Gemüte eine solche Ursache für eine Befriedigung vorfinden; ja, wenn wir nur keinen sehr akuten Schmerz leiden und keiner unmittelbaren Lebensgefahr ausgesetzt sind: so können wir für andere fühlen, auch während wir selbst leiden; und dann oft am meisten, wenn uns das eigene Leid sanfter gemacht hat. Wir sehen mit Mitleid selbst auf solche Nöte, die wir für die eigenen in Tausch nehmen würden." (Burke 1956, 83) Einer der Gründe für eine solche Moderation des Sicherheitsarguments ist im Wandel der politischen und gesellschaftlichen Ordnung zu suchen. Nach Burke steht dem Erhabenen als dem Prinzip einer ,heroischen' Kultur das Schöne als Prinzip eines konsensualen Gesellschaftsmodells gegenüber (vgl. Eagleton 1994). Letzteres ist zwar ästhetisch weniger attraktiv, aber moderner und zeitgemäßer als die archaische Zwangsgewalt des Tyrannisch-Erhabenen, die im ancien régime, in despotischen Systemen oder im alttestamentlichen Gottesbegriff zum Ausdruck kommt (Burke 1956, 93, 99ff.). Das Schöne folgt dagegen den sanfteren Gesetzen des sozialen Zusammenlebens, die im Sinnbild der Mutterliebe konvergieren: „Die Autorität unseres Vaters, so nützlich für unser Wohlbefinden und so verehrungswürdig sie in jeder Hinsicht ist, hindert uns doch daran, zu ihm dieselbe Liebe zu haben wie zu unserer Mutter, bei der die elterliche Autorität fast zerschmilzt in mütterlicher Zärtlichkeit und Nachsicht“ (Burke 1956, 151). Damit beginnt der ästhetische Schrecken - im Gegensatz zum politischen Sprachgebrauch - allmählich eine aktivische Bedeutung (,Schrecken verbreiten') abzulegen und sich auf einen passivischen und rezeptiven Sinn (,Schrecken empfinden') zu beschränken. In den idealistischen Erhabenheitstheorien 
Kants und Schillers findet diese Bewegung, die hier stringenter erscheint, als sie ist, ${ }^{5}$ ihren Höhepunkt und Abschluss. Wir können also zusammenfassen: In dem Maße, in dem Schrecken und Terror als Instrumente einer innenpolitischen Sicherheitsarchitektur geächtet werden, machen sie als ästhetische Begriffe Karriere. Als solche können sie vermöge einer moralisch avancierten Gefühlskultur in eine Quelle der ,negativen Lust' transformiert werden (Kant 1974, 165).

\section{Sicherheit und Freiheit (Hobbes, Schiller)}

Dabei steht die Konjunktur des Sicherheitsarguments in den Kunstphilosophien Kants und Schillers scheinbar reziprok zu Tendenzen des politischen Diskurses. Denn die Einsicht, dass , absolute Sicherheit' nicht herstellbar ist, hindert die idealistische Ästhetik augenscheinlich nicht, eine solche in der subjektiven Innerlichkeit neu zu begründen - im Gegenteil. Schon Hobbes hatte konzedieren müssen, dass das innenpolitische Sicherheitsstreben Leviathans an natürliche Grenzen stößt. Diese hatte Hobbes als Rückzugsraum bürgerlich-individueller Freiheit definiert, wobei Freiheit gleichsam negativ zu einem Ideal der absoluten Sicherheit gefasst wird. Freiheit nach Hobbes ist ein Name für jene Restrisiken, die durch den politischen Souverän nicht ausgeschaltet werden können und deshalb anerkannt werden müssen: „Denn es gibt auf der ganzen Welt keinen Staat, der genügend Vorschriften zur Regelung aller menschlichen Handlungen und Äußerungen erlassen" kann (Hobbes 1984, 165). Deshalb ist Freiheit aber auch mit dem LeviathansApparat der ,Furcht und Notwendigkeit' vereinbar, vorausgesetzt, dass erstere der Sicherheit im Großen und Ganzen nicht schadet. ${ }^{6}$ Sicherheit und Freiheit sind damit freilich oppositorisch aufeinander bezogen. Mit der Aufwertung des Freiheitsbegriffs im 18. Jahrhundert - die durch die von Hobbes verordnete Trennung von politischer und privater Sphäre allerdings begünstigt wird (vgl. Schmitt 1982; Koselleck 1973) - ändert sich dies jedoch. Uns haben an dieser Stelle nur die ästhetischen Implikationen dieses globalen, hier schematisch verkürzten Prozesses zu interessieren. Auch der kunstphilosophische Diskurs kann, wie das Beispiel der Erhabenheitstheorien beweist, die Begriffe von Sicherheit und Freiheit spätestens im 18. Jahrhundert nicht mehr antithetisch konzipieren. Da Sicherheit für eine Ästhetisierung des Schreckens andererseits unabdingbar scheint als conditio sine qua non einer erhabenen Empfindung (vgl. Begemann 1987) - bleiben nur zwei Möglichkeiten: Entweder wird Sicherheit, wie u.a. bei Burke, als physische und ,äußere' Sicherheit verstanden - dann bezeichnet Freiheit lediglich die Abwesenheit von Unsicherheit und wird mit Sicherheit mehr oder weniger identisch („Freiheit von Gefahr"; Burke 1956, 83); oder aber Sicherheit meint, wie v.a. bei Schiller, ,innere' Sicherheit im Sinne einer autonomen Selbstbestimmung des Subjekts - dann wird sie zur Rückund Gegenseite einer universalen, äußeren' Unsicherheit und Freiheit demgegenüber als

5 Carsten Zelle weist z.B. auf eine wichtige Ausnahme in Gestalt von Johann Georg Sulzers Allgemeiner Theorie der schönen Künste von 1794 hin, deren Artikel „Schreken, Schreklich“ die alte Abschreckungsästhetik zustimmend zitiert (Zelle 2003).

6 Die Grenzen der Freiheit sind daher auch durch Furcht und Notwendigkeit festgelegt. Denn: „Niemand hat die Freiheit, dem staatlichen Schwert Widerstand zu leisten [...]“ (Hobbes 1984, 169). 
ultimatives Residuum der Sicherheit bestimmt (,Resignation in die Notwendigkeit“; Schiller 1989, 794). So kann Schiller Burkes Modifikation einer narzisstischen Psychologie à la Hobbes (,joy of grief'; Hobbes 1962, 51f.) zitieren und sie gleichzeitig durch eine Unterscheidung zwischen ,äußerer' und ,innerer', bedingter und unbedingter Sicherheit weiterführen: „Aber dieses Gefühl der Sicherheit bei der Vorstellung fremden Leidens ist ganz und gar nicht der Grund des Erhabenen und überhaupt nicht die Quelle des Vergnügens, das wir aus dieser Vorstellung schöpfen. Erhaben wird das Pathetische bloß allein durch das Bewußtsein unsrer moralischen, nicht unsrer physischen Freiheit. Nicht weil wir durch unser gutes Geschick diesem Leiden entzogen sind (denn da würden wir noch immer einen sehr schlechten Gewährsmann für unsre Sicherheit haben), sondern weil wir unser moralisches Selbst der Kausalität dieses Leidens, nämlich seinem Einfluß auf unsre Willensbestimmung, entzogen fühlen, erhebt es unser Gemüt und wird pathetischerhaben." (Schiller 1989, 510f.)

\section{Innere Sicherheit und ästhetischer Schein bei Schiller}

Schillers Ästhetik des Erhabenen macht also mit dem Schrecken und dem daraus resultierenden Leiden ernst und imaginiert keinerlei materielle Exemtions- und Sicherungsmöglichkeit mehr - bis auf jene „,idealische und über alle möglichen Fälle sich erstreckende Sicherheit, deren wir uns bei der Vorstellung des Erhabenen bewußt werden“ (Schiller 1989, 502). Die Erfahrungshintergründe einer solchen ,innenstabilisierten' Sicherheitsdefinition können hier nicht dargelegt werden (wobei besonders auf die Rolle der Französischen Revolution und einer neostoizistischen Denktradition einzugehen wäre). Fest steht: Weder dem „dynamischen Staat der Rechte“, in dem „Mensch dem Menschen als Kraft begegnet und sein Wirken beschränkt" (Schiller 1989, 667), noch dem ,ästhetischen Staat“, in dem der Mensch frei von Leidenschaften ist, ohne seinen ,sinnlichen Trieb dem vernünftigen unbedingt“ unterzuordnen (Schiller 1989, 607), traut Schiller ein auf Dauer wirksames Sicherheits- bzw. Unsicherheitsmanagement zu. Vielmehr muss das Ideal einer ästhetischen Aussöhnung der Gegeninteressen im Zeichen des Schönen auch wieder durch eine erhabene Selbstrettung des Subjekts abgesichert werden und „das Erhabene zu dem Schönen hinzukommen, um die ästhetische Erziehung zu einem vollständigen Ganzen zu machen und die Empfindungsfähigkeit des menschlichen Herzens nach dem Umfang unsrer Bestimmung, und also auch über die Sinnenwelt hinaus, zu erweitern" (Schiller 1989, 806f.). Ziel einer solchen Sicherheitsstrategie ist nicht die physische „Selbsterhaltung“ gegen die Mächte des Schreckens (wie bei Burke 1956, 72), sondern die paradoxe Apotheose des Selbst im Akt der freiwilligen Unterwerfung seiner Körpernatur. Damit operiert Sicherheit freilich nicht mehr in der Außenwelt. Auch vollzieht Schiller die moderne Umstellung des Sicherheitsbegriffs zu jenem des Risikos - im mathematischen oder, versicherungs'-technischen Sinne - nicht mit. Die Ursachen dafür sind komplex und in der Forschung noch nicht untersucht. Mit verantwortlich für eine solche Haltung dürfte ein epistemologischer Umstand sein: Schiller vermag die Unsicherheit der Zukunft nicht mit Hilfe der Kriterien wahrscheinlich/unwahrscheinlich zu selektieren, weil die Vorstellung der Wahrscheinlichkeit bei ihm für den ästhetischen Diskurs reserviert ist, in dem sie aber nicht das Gegenteil von Unwahrscheinlichkeit noch die stochastische Größe der ,Gleichwahrscheinlichkeit' bezeichnet, sondern durch die Differenz zum ,logischen Schein', also zu Betrug und Täuschung, definiert ist: „Es versteht sich wohl von selbst, daß hier nur von dem ästhetischen Schein [qua Wahrscheinlichkeit] die Rede ist, den man von der Wirklichkeit und Wahrheit unterscheidet, nicht 
von dem logischen, den man mit derselben verwechselt [...]“ (Schiller 1989 656f.). In diesem Sinne referiert ästhetische Wahrscheinlichkeit auf eine „innere Wahrheit“ der Kunst (Goethe 1982, 70), die an der aristotelischen Bestimmung der Wahrscheinlichkeit als dem „nach den Regeln der Wahrscheinlichkeit oder Notwendigkeit Mögliche[n]“ orientiert ist (Aristoteles 1989, 29, 83ff.).

Dabei ist die Wahrscheinlichkeit - oder ,Möglichkeit' - der Welt im Kunstwerk über einen (relativ) stabilen Gegenbezug sowohl zum ,bloß Wirklichen' wie zum ,Zufälligen' konzipiert. Für die Einstellung - etwa der Tragödie - zu den Kategorien von ,Providenz und Kontingenz' und deren gegenseitige Konturierung bleibt das nicht ohne Folgen (vgl. Frick 1988; Graevenitz/Marquard, 1998). In der Sprache der Luhmannschen Systemtheorie könnte man sagen: Kunst macht den Schadensfall zwar beobachtbar (für eine Beobachtung erster Ordnung), ist aber nicht imstande, eine belastbare Aussage hinsichtlich seiner Ereigniswahrscheinlichkeit zu treffen. ${ }^{7}$ Angesichts des bereits eingetretenen Unglücks, das in der Tragödie nicht als wahrscheinlich/unwahrscheinlich, sondern als unvermeidlich und notwendig dargestellt wird, empfiehlt sie dem Beobachter jedoch (so jedenfalls Schiller), sich ,in die unbezwingliche Burg unserer moralischen Freiheit“ zurückzuziehen und dadurch eine ,absolute und unendliche Sicherheit“ zu gewinnen, ,indem wir eine bloß komparative und prekäre Schutzwehre im Feld der Erscheinung verlorengeben" (Schiller 1989, 525). Eine solche Inversion des Sicherheitsbegriffs ist, so die These, von der Medialisierung des Schreckens im modernen Erhabenheitsdiskurs und der Emanzipation einer ästhetischen Theorie der Wahrscheinlichkeit nicht zu trennen. Zwar kennt auch die mittelalterliche und frühneuzeitliche Tradition der certitudo eine strikte Entgegensetzung von (innerer) Glaubensgewissheit und (äußerer) Sicherheitsillusion (vgl. Schrimm-Heins 1990/91). Aber anders als die idealistische Ästhetik traut sie dem Kunstwerk in diesem Punkt keine eigenständige Wahrheitsmöglichkeit oder Erkenntniskompetenz zu, weshalb noch in der barocken Poetik das ,Spiel' des Trauerspiels (als künstliches Unglück) mit dem des theatrum mundi (als tatsächlichem Unglück) zusammenfällt, statt einen separaten Diskurs zu begründen. ${ }^{8}$ Für ein zugleich innerweltliches und enttäuschungsresistentes Konzept der Sicherheit dürfte daher die kulturelle Evolution eines Sicherheitsgefühls in den ästhetischen (und philosophischen) Reflexionen des 18. und 19. Jahrhunderts maßgeblich von Bedeutung sein.

7 Andererseits betont Rüdiger Campe die gedankliche Verwandtschaft des probabilistischen Wahrscheinlichkeitskalküls der frühen Neuzeit mit traditionellen Vorstellungen der Wahrscheinlichkeit in Rhetorik und Poetik (vgl. Campe 2002). Darauf kann hier, wo es lediglich um den Aufriss eines Forschungsfeldes geht, nicht näher eingegangen werden.

8 Dies wird etwa in der berühmten Widmungsvorrede zu Daniel Caspar Lohensteins Trauerspiel Sophonis$b e$ (von 1680) deutlich, in dem es programmatisch - und ohne Differenzierung zwischen einem ästhetischen und lebensweltlichen Spiel- und Wahrscheinlichkeitsbegriff - heißt: „Für allen aber ist der Mensch ein Spiel der Zeit. / Das Glücke spielt mit ihm / und er mit allen Sachen“ (Lohenstein 1991, 8; vgl. auch Voßkamp 1967). 


\section{Postheroische Gelassenheit}

Dies bestätigt auch die aktuelle politische und sozialwissenschaftliche Diskussion über symmetrische und asymmetrische Sicherheitsarchitekturen. Folgt man ihr, so sind es im Wesentlichen zwei Zukunftsszenarien, die angesichts der ,neuen' Sicherheitslage nach dem 11. September 2001 wahrscheinlich werden: Entweder es gelingt den postheroischen Gesellschaften des Westens sich sicherheitstechnisch an die Herausforderungen des transnationalen Terrorismus zu adaptieren - oder ,es entwickelt sich in der Bevölkerung eine postheroische Gelassenheit, die auch durch längere Anschlagsserien nicht zu erschüttern ist und an der die terroristischen Anschläge schließlich abprallen. Heroische Gelassenheit der Bevölkerung panzert nämlich das eigentliche Angriffsziel des transnationalen Terrorismus: die labile psychische Infrastruktur postheroischer Gesellschaften " (Münkler 2006, 343). Ein solches Revirement heroischer Verhaltenstugenden müsste sich in Zeiten einer postheroischen Demobilisierung des kulturellen Gedächtnisses der Kunst bedienen, in dem entsprechende Reaktionsweisen gespeichert und psychosozial konditioniert worden sind. ${ }^{9}$ Damit würde aber - unter ganz anderen historischen und gesellschaftlichen Vorzeichen - eine Konstellation der klassischen Ästhetik des Erhabenen wiederkehren, wie sie hier exemplarisch an Schiller demonstriert worden ist: die einer selbstgewissen Duldung lebensweltlicher Sicherheitsfrustrationen, die letztlich von einer moralischen Überlegenheitsgewissheit getragen ist. ${ }^{10} \mathrm{Zu}$ den Erfolgschancen eines solchen Akts der mémoire volontaire (der sich auf das Erinnerungspotenzial der Bilder und Texte zu stützen hätte) kann man gegenwärtig noch wenig sagen. Sicher aber ist, dass das damit unterstellte intuitive Selbstsicherungswissen entscheidend von ästhetischen Denkmustern geprägt ist. Damit wird einerseits die Funktion künstlerischer Sicherheitsexperimente für den psychologischen Haushalt des postheroischen Individuums deutlich und andererseits die Geschichtlichkeit eines Sicherheitsbedürfnisses transparent, das von den Umbauphasen der modernen Sicherheitstechnik scheinbar unabhängig ist.

\section{Bibliographie}

Aristoteles (1989) Poetik. Griechisch/Deutsch. Stuttgart: Reclam.

Barone, P. (2004) Schiller und die Tradition des Erhabenen. Berlin: Erich Schmidt.

Begemann, C. (1987) Furcht und Angst im Prozeß der Aufklärung. Zu Literatur und Bewußtseinsgeschichte des 18. Jahrhunderts. Frankfurt a.M.: Athenäum.

Blumenberg, H. (1998) Terminologisierung einer Metapher: ,Wahrscheinlichkeit'. In: Blumenberg, H. Paradigmen zu einer Metaphorologie. Frankfurt a.M.: Suhrkamp: 117-141.

9 Die moderne Risikogesellschaft, die die latente Unsicherheit der Sicherheit immer schon in ihren Sicherheitsbegriffs mit eingebaut hat, bedarf des Schemas des Heroischen oder Erhabenen dagegen nicht - wie Niklas Luhmann konstatiert: „Die Risikogesellschaft kennt keine Helden und keine Herren“ (Luhmann 1991, 113).

10 Dabei wäre die Rolle des heroischen Paradigmas für eine Theorie des Erhabenen bei Schiller einerseits und bei Burke andererseits jeweils unterschiedlich zu bewerten (vgl. dazu auch Barone 2004, und Bruyn 1987). 
Bonß, W. (1995) Vom Risiko. Unsicherheit und Ungewißheit in der Moderne. Hamburg: Hamburger Edition.

Bredekamp, H. (2006 [2003]) Thomas Hobbes. Der Leviathan. Das Urbild des modernen Staates und seine Gegenbilder, 1651-2001. Berlin: Akademie-Verlag.

Bruyn, F. de (1987) Hooking the Leviathan. The Eclipse of the Heroic and the Emergence of the Sublime in eighteenth-century British Literature. In: The Eighteenth-Century: 28/3: 195-215.

Buldt, B. et al. (2004) Wahrscheinlichkeit. In: Ritter, J./Gründer, K./Gabriel, G. (Hg.) Historisches Wörterbuch der Philosophie. Basel: Schwabe: vol. 12: 251-304.

Burke, E. (1956 [1757]) Vom Erhabenen und Schönen. Berlin: Aufbau.

Burke, E. (1987 [1790]) Betrachtungen über die Französische Revolution. Zürich: Manesse.

Campe, R. (2002) Spiel der Wahrscheinlichkeit. Literatur und Berechnung zwischen Pascal und Kleist. Göttingen: Wallstein.

Conze, W. (1994) Sicherheit, Schutz. In: Brunner, O./Conze, W./Koselleck, R. (Hg.), Geschichtliche Grundbegriffe. Historisches Lexikon zur politisch-sozialen Sprache in Deutschland. Stuttgart: Klett-Cotta: vol. 5: 831-862.

Eagleton, T. (1994 [1990]) Ästhetik. Die Geschichte ihrer Ideologie. Stuttgart: Metzler.

Endres, J. (2008) Sicherheit als kulturelles Paradigma. Hobbes, Burke, Schiller und die Theorie des Erhabenen. In: Petersen, C./ Riou, J. (Hg.) Zeichen des Krieges in Literatur, Film und den Medien. Bd. 3: Terror. Kiel: Verlag Ludwig (im Erscheinen).

Foucault, M. (2004) Geschichte der Gouvernementalität. Vorlesung am Collège de France 1977-1978/1978-1979. Hg. von M. Sennelart. vol. 2, Frankfurt a.M.: Suhrkamp.

Frick, W. (1988) Providenz und Kontingenz. Untersuchungen zur Schicksalssemantik im deutschen und europäischen Roman des 17. und 18. Jahrhunderts, Tübingen: Niemeyer.

Goethe, J. W. (1982[1798]) Über Wahrheit und Wahrscheinlichkeit der Kunstwerke. Ein Gespräch. In: Goethe, J. W. Werke. Hamburger Ausgabe. Hg. von E. Trunz. München: Deutscher Taschenbuchverlag: vol. 12: 67-73.

Graevenitz, G. v./Marquard, O. (Hg.) (1998) Kontingenz. München: Fink.

Hobbes, T. (1966 [1658/1647]) Vom Menschen. Vom Bürger (Elemente der Philosophie II/III. Hg. von G. Gawlick. Hamburg: Meiner.

Hobbes, T. (1991 [1651]) Leviathan oder Stoff, Form und Gewalt eines kirchlichen und bürgerlichen Staates. Frankfurt a.M.: Suhrkamp.

Kant, I. (1974 [1790]) Kritik der Urteilskraft. In: Kant, I., Werkausgabe. Hg. von W. Wieschedel. Frankfurt a.M.: Suhrkamp.

Kaufmann, F.-X. (1973 [1970]) Sicherheit als soziologisches und sozialpolitisches Problem. Untersuchungen zu einer Wertidee hochdifferenzierter Gesellschaften. Stuttgart: Ferdinand Enke.

Koselleck, R. (1973 [1959]) Kritik und Krise. Eine Studie zur Pathogenese der bürgerlichen Welt. Frankfurt a.M.: Suhrkamp.

Lohenstein, D. C. (1991 [1680]) Sophonisbe. Trauerspiel. Hg. von R. Tarot. Stuttgart: Reclam.

Luhmann, N. (1991) Soziologie des Risikos. Berlin/New York: de Gruyter.

Münkler, H. (2006) A New Security Architecture. In: Internationale Politik (IP) 2(8): 2835.

Münkler, H. (2007) Der Wandel des Krieges. Von der Symmetrie zur Asymmetrie. Weilerswist: Velbrück Wissenschaft. 
Schiller, F. (1989) Sämtliche Werke. Hg. von G. Fricke u. H. G. Göpfert. München: Carl Hanser: vol. 5.

Schmitt, C. (1963) Theorie des Partisanen. Zwischenbemerkungen zum Begriff des Politischen. Berlin: Duncker und Humblot.

Schmitt, C. (1982 [1938]) Der Leviathan in der Staatslehre des Thomas Hobbes. Sinn und Fehlschlag eines politischen Symbols. Stuttgart: Klett-Cotta.

Schrimm-Heins, A. (1991/92) Gewissheit und Sicherheit. Geschichte und Bedeutungs-

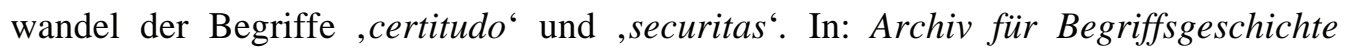
34/35: 123-213/115-213.

Voßkamp, W. (1967 [1965]) Zeit- und Geschichtsauffassung im 17. Jahrhundert bei Gryphius und Lohenstein. Bonn: Bouvier.

Walther, R. (1990) Terror, Terrorismus. In: Brunner, O./Conze, W./Koselleck, R. (Hg.) Geschichtliche Grundbegriffe. Historisches Lexikon zur politisch-sozialen Sprache in Deutschland. Stuttgart: Klett-Cotta: vol. 6: 323-444.

Zelle, C. (1987) Angenehmes Grauen. Literaturhistorische Beiträge zur Ästhetik des Schrecklichen im achtzehnten Jahrhundert. Hamburg: Meiner.

Zelle, C. (2003) Schrecken, Schreck. In: Barck, K. (Hg.) Ästhetische Grundbegriffe. Historisches Wörterbuch in sieben Bänden. Stuttgart/Weimar: Metzler: vol. 5: 436-446.

Johannes Endres, Promotion 1995 (Universität Trier), Habilitation 2004 (Universität Leipzig); seit 2007 Lehrkraft für besondere Aufgaben (Universität Göttingen); Forschungsschwerpunkte: Literatur und Kultur um 1800; Topoi und Metaphern; Bild und Text.

E-mail: Johannes.Endres@phil.uni-goettingen.de 